\title{
Mapas de contenidos para una mayor implicación y autonomía del alumno
}

\section{Content maps as a tool for increasing student's involvement and autonomy}

ANA BELÉn FERIA BOURRELLIER

ORCID: https://orcid.org/ 0000-0003-1532-5601

Departamento de Biología Vegetal y

Ecología, Área de Fisiología Vegetal

Facultad de Biología

Universidad de Sevilla

anabelen@us.es.

Fecha de recepción: 19-11-2019

Fecha de aceptación:

DOI: http://dx.doi.org/10.12795/9788447221912.082

Pp.: 1870-1888 


\section{Resumen}

Mi objetivo en este ciclo de mejora en el aula (CIMA) ha sido la utilización del mapa de contenidos como herramienta principal en la enseñanza-aprendizaje de una disciplina clave en el marco de Europa 2020 (Horizonte 2020), fisiología vegetal. Entre sus objetivos y prioridades, aparecen la biotecnología, seguridad alimentaria, agricultura sostenible, energía limpia, segura y eficiente y cambio climático. La fisiología vegetal está estrechamente relacionada con todos ellos. Los cuatro grandes bloques que desarrollamos son: nutrición, metabolismo, crecimiento y desarrollo y estrés de la planta. Los contenidos que deben aprender los alumnos quedan recogidos en estos mapas, donde queda establecida la relación de cada pregunta clave con la parte del mismo que pretende abarcar. Este modelo metodológico da valor a los saberes previos de los estudiantes ajustando el diseño a su diversidad y activando sus dimensiones cognitivas y emocionales.

Palabras clave: Fisiología Vegetal, Grado en Bioquímica Docencia universitaria, "xperimentación docente universitaria, Aprender Investigando.

\section{Abstract}

Within this cycle of improvement in the classroom (CIMA) my main objective has been the use of the content mapping. Content mapping is a major tool for the teaching-learning process of plant physiology, a key discipline within the Europe 2020 program (Horizon 2020). Plant physiology treats many important and socially relevant issues such as biotechnology, food security, sustainable agriculture, clean, safe and efficient energy, green transport and climate change. Plant physiology is closely related to all of them. The four big blocks we develop here are: nutrition, metabolism, growth and development and abiotic and biotic stress of the plant. For the students it is essential to understand the content maps which represent the relationship of each key issue and associated motivating questions. In addition, all contents are developed using data, concepts, examples or procedures. Using the technique of content mapping focusing on the student's problems when a approaching a new subject, we intend to mimic the students' own mental schemes. In my own practice as a teacher this methodological model turned out to be very useful. The model gives value to the a priori opinions and the a priori knowledge of the students dynamically adapting to their diverse knowledge and interest.

Key words: "Plant Biology", "Degree in Biochemistry", "University teaching", "University teaching experimentation", "Learning by Investigating". 


\section{Descripción del Contexto de la Intervención}

La asignatura donde se lleva a cabo la intervención es "Fisiología Molecular de Plantas". El programa de la asignatura centra su atención en la identificación molecular y la función de los componentes, estructuras y órganos que intervienen en la fisiología de la planta desde una perspectiva integrada, focalizándose en las plantas vasculares.

La asignatura es una troncal del primer cuatrimestre, obligatoria de tercer curso del Grado de Bioquímica que imparte la Facultad de Biología en la Universidad de Sevilla. Los créditos totales de la asignatura son 6, 4 de teoría y 2 de prácticas. Posee 45 alumnos matriculados en un solo grupo, y las clases las reciben 3 horas a la semana repartidas en 3 días. Las clases teóricas se imparten por medio de clases magistrales en las que el profesor destina un tiempo a fomentar la participación del alumno mediante sesiones de seminarios, cuestiones, debates y resolución de problemas. En cada clase, el profesor apoya la explicación diaria con proyecciones de transparencias, diapositivas o videos que, en la medida de lo posible, se encuentran a disposición de todos los alumnos en la plataforma de enseñanza virtual de la Universidad de Sevilla.

Los objetivos que se han planteado han sido: a) Objetivos conceptuales: Conocimiento de las características fundamentales de las plantas, autotrofia, fotosíntesis, absorción del agua y de los nutrientes. Conocimiento de la estructura y organización de la planta y de su crecimiento y desarrollo, regulación de los procesos e interacción con el medio; b) Objetivos procedimentales: Manejo de medios técnicos y equipos básicos de laboratorio, análisis de datos y resolución de problemas planteados en la experimentación con plantas. Redacción y exposición de resultados, en relación con la información bibliográfica actualizada y c) Objetivos actitudinales: Desarrollo de la capacidad de autoaprendizaje, disposición al trabajo en equipo, capacidad 
de análisis y crítica de contenidos científicos y de resultados experimentales.

Los problemas que me surgen como docente es que el programa abarca muchos contenidos en un breve espacio de tiempo; por ello, he de hacer una selección de contenidos con todas las inseguridades que se derivan de ello. Aunque tengamos la creencia generalizada de que los programas han de impartirse en su totalidad, ya que éstos reflejan la mejor síntesis de la disciplina, ni todo el conocimiento de la disciplina es igualmente importante, ni hay un único nivel de formulación correcto. El problema surge cuando elaboras los conocimientos en forma de listados, en ese caso si eliminas un eslabón puede ser una carencia importante, pero si se construye todo un entramado, eliminar pequeñas piezas, no debería desencajarlo. Toda la disciplina es un conjunto de contenidos organizados, jerarquizados y conectados que pueden adoptar diferentes versiones. ¿Y que eslabón no eliminar?, es la pregunta consecuente, pues, contenidos que respondan a problemas actuales, relevantes a día de hoy, y no, aquellos que surgieron como respuesta a problemas pasados. Hay que repensar los contenidos. La descripción de contenidos que ni siquiera están en vanguardia deben ser sustituidos por contenidos y dilemas innovadores.

\section{Principios Didácticos}

El principio didáctico que da nombre a esta intervención es el uso de un mapa de contenidos en el que quede establecida claramente la relación de cada pregunta-clave con la parte del mismo que puede abarcar. El principio didáctico que más se trabaja en este CIMA es el que los estudiantes son capaces de plantearse preguntas que son nexos con los contenidos, si los formulamos como problemas. Lo primordial que me planteo es que quede claro cuál es el concepto principal del bloque temático y como 
se relaciona éste con los conceptos principales de los otros bloques, haciéndoles entender que la fisiología vegetal, a diferencia de la bioquímica pura, es una ciencia integradora que intenta estudiar el funcionamiento general de la planta.

Las competencias a desarrollar será que el estudiante integre los conceptos bioquímicos, moleculares y fisiológicos, para comprender como afectan al funcionamiento general de la planta. Que no se queden en el funcionamiento de una enzima en concreto, sino que sean capaces de integrarlo con el metabolismo general de la planta, y como una disfunción puntual puede alterar finalmente el desarrollo de la planta y su relación con el medio.

\section{Mapas de Contenidos y Preguntas Clave}

Los contenidos también son elementos susceptibles de mejora cuando queremos cambiar la enseñanza a pesar de que en lo primero que se piense es en la metodología ó en el sistema de evaluación (García-Díaz, Porlán y Navarro, 2017). Los contenidos a enseñar rara vez nos los planteamos, pues nos llegan impuestos ya que pasaron por la memoria recogida en el programa que se envía en la verificación del Grado, y asumimos que son incuestionables, Además, por otra parte, de ser muy cómoda dicha imposición, para no tener que plantearnos también el porqué de su enseñanza. Así terminamos utilizando diferentes recursos dependiendo del contenido a enseñar, pero intentando transmitir absolutamente todo nuestro conocimiento. Finalmente, el propio programa termina siendo la principal barrera para el cambio, por lo que la revisión del programa debe ser una de nuestras labores docentes para preparar a nuestros estudiantes para una nueva demanda laboral con nuevos retos profesionales que deben ser apoyados por contenidos acordes a ellos. Nosotros como expertos en educación debemos ser capaces de replantearnos los temarios y enfrentarnos a nuestra propia ignorancia sobre la disciplina. 
Dado que el tiempo es limitado, ha sido necesario seleccionar los contenidos más relevantes, pero siempre estableciendo relaciones de significado entre ellos. Los contenidos quedan así formulados y organizados en mapas. Se distinguen los tipos de contenidos y se construye la interacción que haya entre ellos, y jerarquizándolos, con el objetivo de que cada alumno haga su propio esquema mental. Según el modelo metodológico que sigo y que resumiré posteriormente, el alumno debe estar implicado activamente en la resolución de problemas relevantes desde el punto de vista de la asignatura. Los contenidos que recogen estos mapas resolverán estos problemas que serán desencadenantes de la construcción del conocimiento de los estudiantes. Quedan así clasificados todos los contenidos a desarrollar en datos, conceptos, ejemplos o procedimientos; así como las prácticas de la asignatura, que se realizarán asociadas a cada uno de los bloques.

Recogiendo los contenidos en mapas que incorporan la formulación de Problemas, pretendo favorecer la expresión de los propios esquemas mentales de los alumnos, quedándose así establecido mi Modelo Metodológico, modelo que me está resultando muy útil en la práctica docente. Este modelo da valor a las opiniones y saberes previos que traen los estudiantes ajustando el diseño a su diversidad y activando dimensiones cognitivas y emocionales de los estudiantes.

Los mapas de contenidos serán 5 (Figuras 1-5), uno primero que intenta relacionar los bloques entre sí. Y otros cuatro, uno por cada bloque temático que desarrollaré y que comprende la asignatura entera: Nutrición Vegetal, Metabolismo primario y secundario de las plantas, Desarrollo y Crecimiento vegetal y Estrés abiótico y biótico de las plantas. Además, estos mapas incorporarán las prácticas asociadas a cada bloque de la asignatura. Es importante que el alumno sitúe la utilidad de cada práctica en su contexto para que le ayude a darle un futuro uso en los diferentes retos que se les presenten en su vida profesional. 


\section{ANA BELÉN FERIA BOURRELLIER}
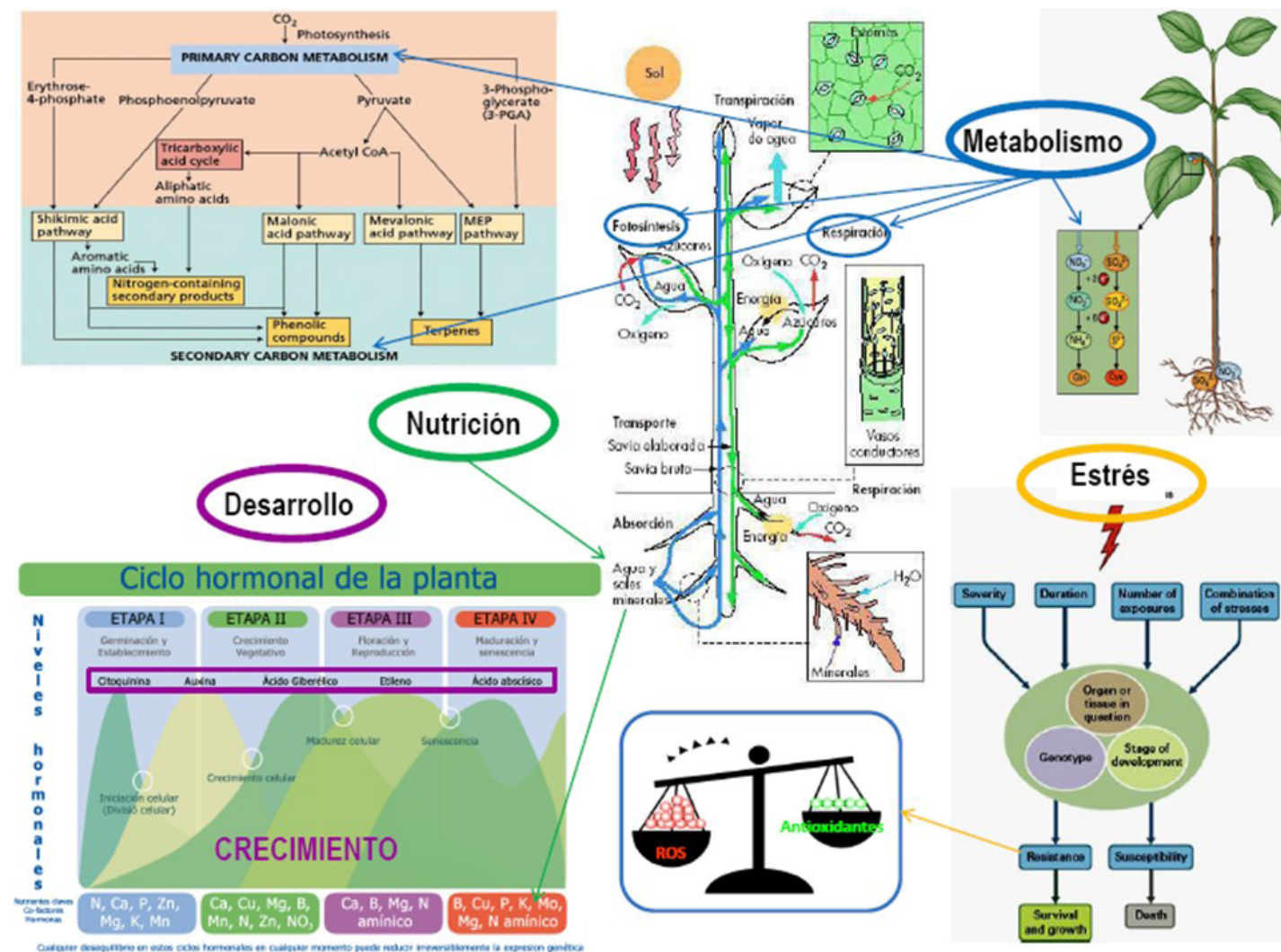

Figura 1. Mapa conceptual general que recoge los grandes bloques que abarca la disciplina

Jornadas de Formación e Innovación Docente del Profesorado | № 2 (2019) Esta obra se distribuye con la licencia Creative Commons Reconocimiento-NoComercial-SinObraDerivada Internacional (CC BY-NC-ND 4.0.) 


\section{ANA BELÉN FERIA BOURRELLIER}

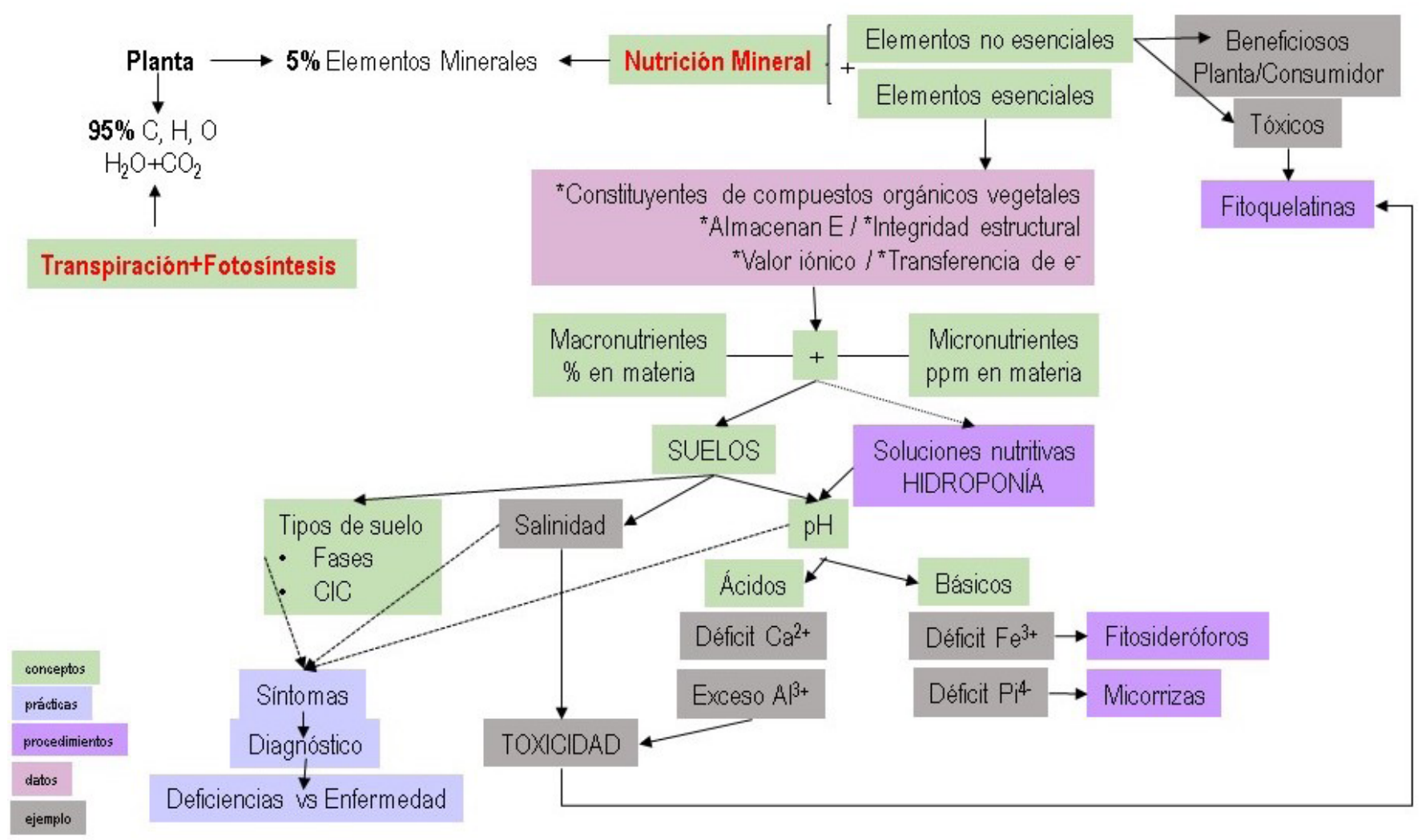

Figura 2. Mapa de contenidos del bloque de Nutrición Mineral Vegetal.

Jornadas de Formación e Innovación Docente del Profesorado I № 2 (2019) Esta obra se distribuye con la licencia Creative Commons

Reconocimiento-NoComercial-SinObraDerivada

Internacional (CC BY-NC-ND 4.0.) 


\section{ANA BELÉN FERIA BOURRELLIER}

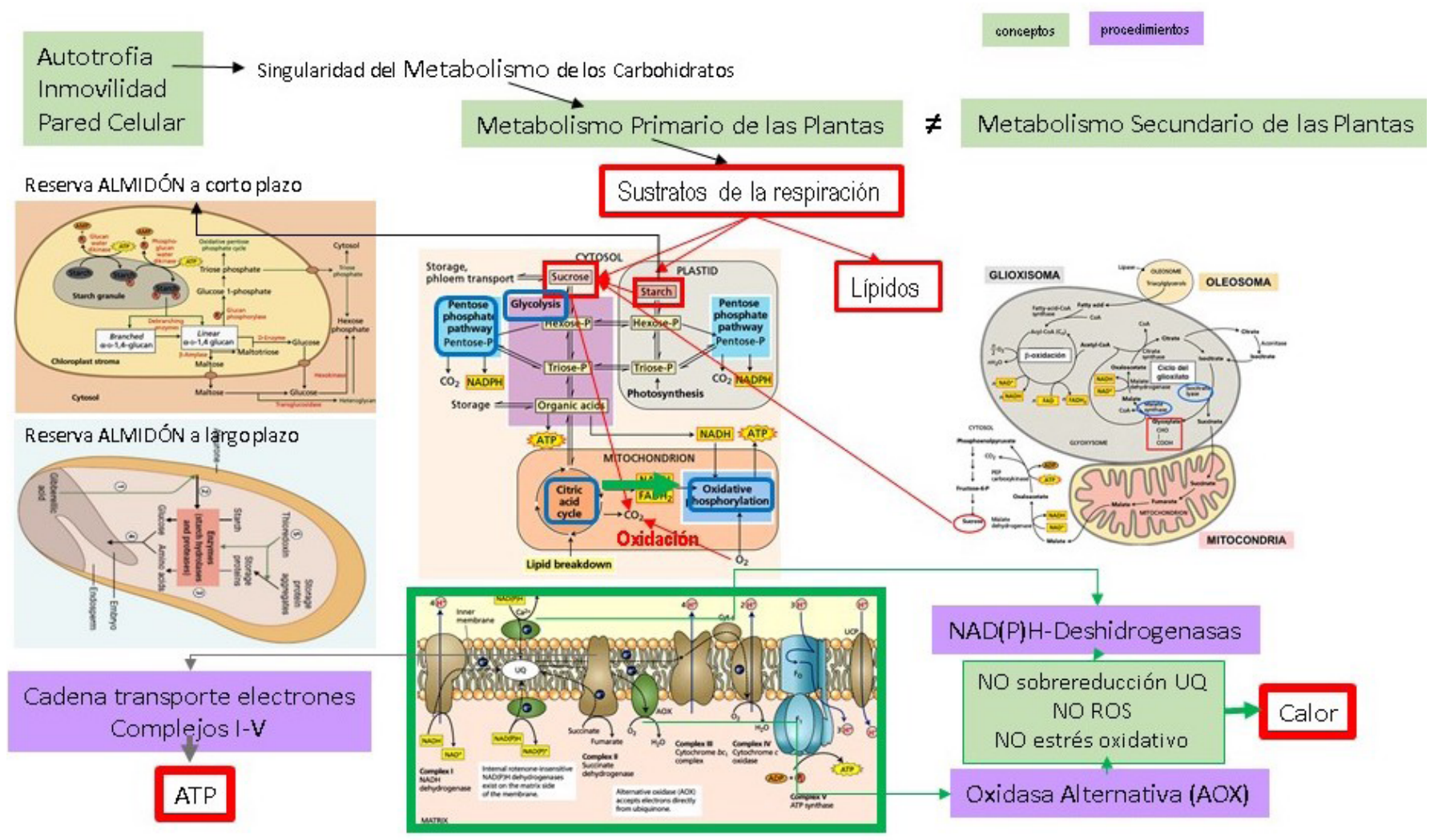

Figura 3. Mapa de contenidos del bloque de Metabolismo de los Carbohidratos.

Jornadas de Formación e Innovación Docente del Profesorado | № 2 (2019) Esta obra se distribuye con la licencia Creative Commons Reconocimiento-NoComercial-SinObraDerivada Internacional (CC BY-NC-ND 4.0.)

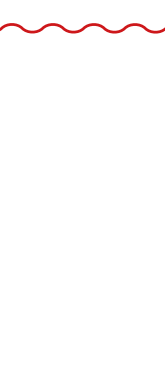


ANA BELÉN FERIA BOURRELLIER

CRECIMIENTO de la planta $\rightarrow \rightarrow \rightarrow$ HORMONAS VEGETALES $\equiv$ FITOHORMONAS

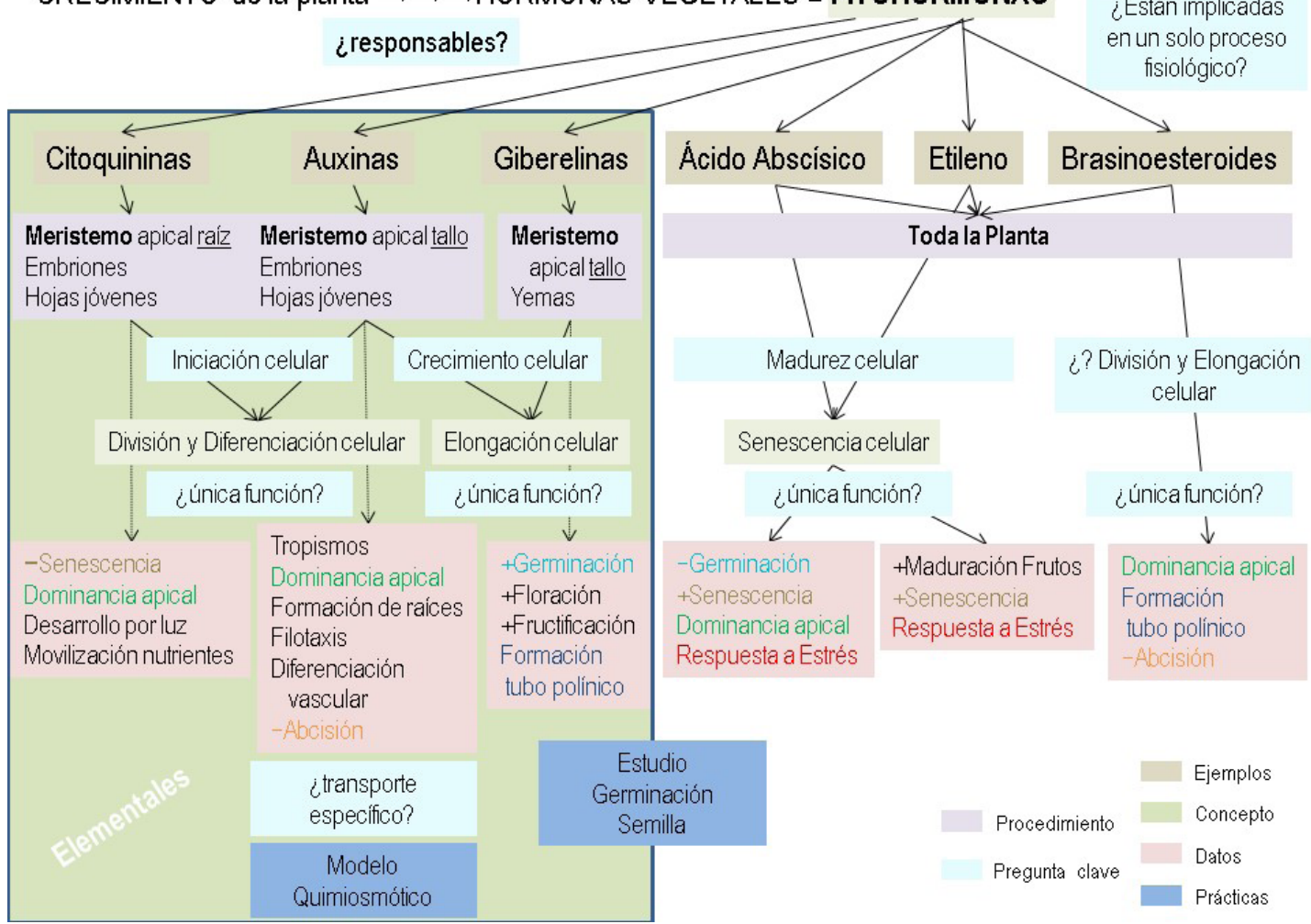

Figura 4. Mapa de contenidos del bloque de Desarrollo, en el se explica el crecimiento de la planta en base a sus ciclos hormonales.

Jornadas de Formación e Innovación Docente del Profesorado I № 2 (2019) 


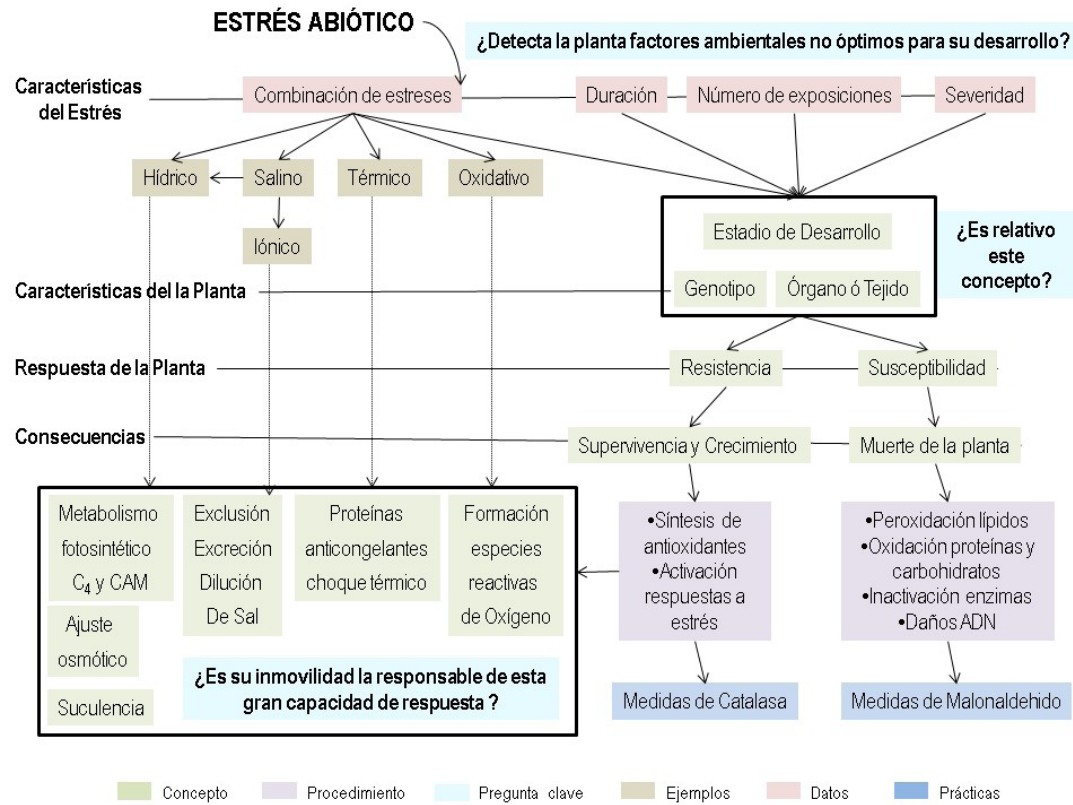

Figura 5. Mapa de contenidos del bloque de Estrés, en él se recogerán los contenidos sobre la fisiología de estrés abiótico en plantas. Imágenes tomadas de los libros Plant Physiology and Development 6aed y Biochemistry and Molecular Biology of Plants 2a ed.

\section{Modelo Metodológico Personal}

El modelo metodológico base que he perseguido ha sido el de "Aprender Investigando" (Travé et al., 2006), iniciando así al alumno en el pensamiento científico (de Alba y Porlán, 2017). En realidad, es la manera natural de trabajar, pues en nuestra carrera investigadora es así como funcionamos; nuestro yo docente, debe retroalimentarse de nuestro yo investigador. El modelo aquí resuelto plantea un problema relevante de la asignatura, escucha las ideas y modelos que proponen los estudiantes y lo llevan a la práctica apoyados por la teoría del profesor (Figura 6) (Feria, 2017). Se establece asi una conexión teórico-práctica de la producción del conocimiento disciplinar (P-IA-T).

Jornadas de Formación e Innovación Docente del Profesorado | № 2 (2019) Esta obra se distribuye con la licencia Creative Commons Reconocimiento-NoComercial-SinObraDerivada 

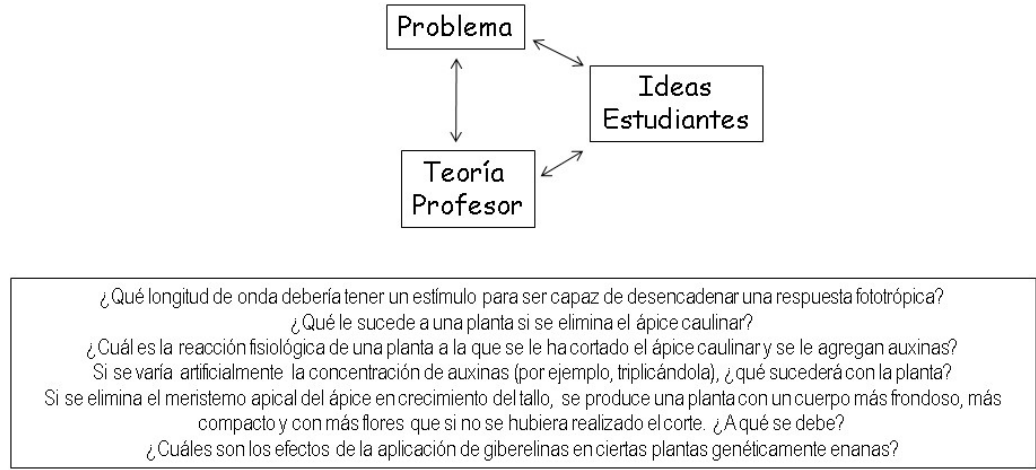
¿Cuáles son los efectos de la aplicación de giberelinas enciertas plantas genéticamente enanas?

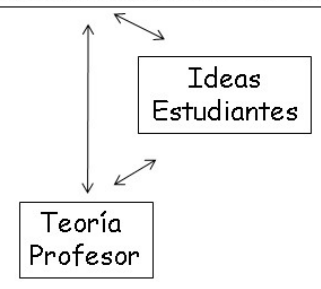

Figura 6. Modelo Metodológico. Modelo que se repite para cada uno de los conceptos abordados. Se realiza un análisis teórico-práctico del problema, con el fin de ir elaborando soluciones. Modelo elaborado para el bloque de

Desarrollo. Preguntas tomadas del libro "Biología” 7a Edición de Curtis.

Retener información, no es aprender; mostrar interés y emoción, no es aprender; el aprendizaje conlleva un proceso que implica saber relacionarlo a otro caso. Para que haya aprendizaje tiene que haber no solo un estímulo y encontrarse receptivo sino una construcción progresiva, una maduración y una relación. Para que el aprendizaje sea perdurable hay que conectarlo muy bien al conocimiento.

Con este modelo se recogen las ideas de los estudiantes, que en contra de la creencia de que hay que corregirlas, lo que se hace es reconducirlas; si asumes que el alumno no tiene nada que aportar es posible que se pierda por el camino. El estudiante es un sujeto que aprende, pero no que se rellena con el conocimiento que yo estoy volcándole, ellos tienen ideas previas, de hecho, no se 
podría aprender si no es a partir de lo que se sabe, y para aprender deben establecer conexiones significativas en un proceso interactivo entre su mente interior y una nueva información que le llega del exterior. Es todo un proceso de construcción de ideas cada vez más complejas de un contenido desde esa simple inicial. Proceso que en la literatura llaman evolución de ideas previas (Sánchez-Romero y Navarro, 2018). En este proceso de construcción del conocimiento del modelo investigativo el docente es una fuente relevante de información. En algunos casos concretos en el desarrollo de este CIMA se ha podido sustituir al docente como fuente de información por fuentes bibliográficas e incluso por una práctica que ayuda a responder a la pregunta clave. Respecto a esta última no se le aporta al alumno todo el conocimiento, pero si se le dan las herramientas para que controlen su propio proceso de aprendizaje dándoles una capacidad auto cognitiva.

\section{Secuencia de actividades}

Este siempre resulta el apartado más controvertido, cómo diseñar una secuencia de actividades que sea coherente con el modelo metodológico aquí presentado y así acometer el problema central por el que se realiza un ciclo de mejora.

La idea es la de ir desglosando el mapa conceptual. Cada clase comenzará abordando un tema principal, por ejemplo si la planta es capaz de detectar factores externos medioambientales que no resultan del todo óptimos para su desarrollo; SI/No pero continuaré preguntando ¿pero de qué depende?....esperando que los alumnos hagan una lluvia de ideas que iré reconduciendo hacia las respuestas correctas y en las que finalmente iremos formando entre todos el mapa conceptual. Ellos dispondrán del material en diapositivas subidas a la plataforma virtual que una vez enlazados con las preguntas clave que se formularán 
en clase sabrán enlazar mentalmente dichas diapositivas. A continuación, describo el relato de una de las sesiones del bloque de desarrollo y crecimiento.

\title{
Relato resumido de las sesiones
}

\author{
Pregunta clave \\ ¿Qué le sucede a la planta si se le elimina el ápice \\ caulinar? \\ Ideas de los \\ Dejaría de crecer. Perdería la percepción de la gravedad. \\ estudiantes \\ No enraizaría... Los alumnos plantean sus ideas y yo \\ las voy rebatiendo, confirmando o ampliando. \\ Experimentos \\ La forma más deseable para que respondieran a esta \\ pregunta es que efectivamente cada uno eliminara \\ Resultados \\ el ápice ó cofia de la raíz y esperara, pero no se \\ dispone de ese tiempo, ni material para repartir a \\ todo el alumnado, ni el aula es el mejor espacio para \\ realizarlo. Aun así, les animo que en casa experimenten \\ con problemas tan básicos como éste. \\ Otra parte de la clase la pasan consultando fuentes \\ bibliográficas. Revisiones que adjunto en el espacio \\ virtual de la asignatura y a la que ellos tienen acceso \\ desde sus dispositivos móviles. \\ Finalmente elaboramos una lista de la cantidad de \\ funciones fisiológicas que pierde la planta al perder \\ parte de este órgano y las repercusiones para su \\ desarrollo e incluso sus aplicaciones en agricultura.
}

\section{Evaluación del Aprendizaje del Alumno}

Se ha seguido un sistema de evaluación clásico por calificación individual llevada a cabo por el profesor. Si bien las últimas corrientes apuntan que se trataría de un aprendizaje por sumisión, en el que se ve la calificación como un soborno y un intento de controlar las clases, aún la maduración de mi CIMA no ha llegado a distinguir entre evaluación y calificación.

¿Cómo es posible medir realmente el aprendizaje de un alumno?, la bibliografia de autores especialistas en 
evaluación (Álvarez-Méndez, 2001) nos recomienda que cuantos mas instrumentos tengamos, y no uno sólo, como podría ser un examen final, más rigurosa será dicha evaluación. Por ello la evaluación global de la asignatura, aprovechando que dispongo de diferentes fuentes que me informan de cómo está ocurriendo el aprendizaje de mis alumnos, se repartirá entre:

1) El trabajo de clase, que ha supuesto bastante tiempo para el alumno. Dicho trabajo será evaluado con la entrega de los mapas. En general los mapas son esquemas de pensamiento, experiencias y valores que van a influir en la manera de reflexionar sobre el tema y representar los contenidos por parte de cada alumno, por lo que, sobre un mismo tema, podrían construirse distintos mapas conceptuales igual de válidos y correctos. Por experiencia, la diversidad no es tal, pues al haber ido reconduciendo la clase con esa interacción constante entre el problema, las ideas de los alumnos, y la teoría del profesor los mapas quedan bastante parejos centrándose en los contenidos principales tratados.

2) Se valorarán las prácticas, es decir las habilidades que también posee el alumno. Tanto su forma de manipular como de interpretar un protocolo de laboratorio. Además, se le puntuará la elaboración de un cuaderno de prácticas en el que discutirá los métodos seguidos y los avalará con bibliografia de nuestra base de datos, publicadas desde principios de curso y a las que ellos tienen acceso.

3) Por último, otro porcentaje de la nota será el correspondiente a un examen escrito final correspondiente a toda la asignatura. Las preguntas formuladas intentarán alejarse lo más posible de la fórmula clásica de enumeración de datos. Es decir, usando este modelo metodológico no preguntaría Mecanismo de acción de 
las giberelinas, pregunta tal cual, de un libro de texto, sino que preguntaría por ejemplo ¿Cuáles son los efectos de la aplicación de giberelinas a plantas que son genéticamente enanas? Siempre preguntas cortas de desarrollo que no se resuelvan con respuestas literales del libro, sino que tengan que relacionarlas con el origen de las cosas. Este examen, aunque es común a todos los estudiantes, en un intento de garantizar el mismo tratamiento a todos, incluye la valoración de cada estudiante individualmente, pues no se sobrevalora unas capacidades sobre otras; son igualmente valoradas capacidades de redacción o esquematización.

He seguido dando mis clases convencionales en las que abordamos todo el contenido de la asignatura, pero con un modelo metodológico que te aleja del dictado de apuntes y la enumeración de datos. Los alumnos tienen la opción de realizar o no los mapas como actividad, incluso pueden descolgarse y todos los que hayan hecho hasta ese momento se les evaluará sumándolo al examen; así no se excluye a ningún alumno que no quiera acogerse a este sistema de evaluación.

\section{Evaluación del Ciclo de Mejora}

Este CIMA analiza el cambio de los contenidos y la metodología, dos de los tres grandes ítems didácticos, faltaría una evaluación más acorde que le diera coherencia y cerrara el conjunto. Tendría que establecer un sistema de evaluación verdadero, una medida correcta de los saberes del alumno, en el que el pueda salirse de interpretar el papel de contar sólo lo que el profesor quiere escuchar. 
La pregunta que queda para mi siguiente ciclo de mejora es: si el mapa es el temario y el mapa es el problema, ¿cómo saber valorar los conocimientos útiles y relevantes que el alumno ha adquirido del mapa? Para contestar a esta pregunta tendré que partir de la base de dos argumentos didácticos: Que el objetivo de la evaluación es la mejora y que hay que ser coherente como se han enseñado los contenidos y la manera de evaluarlos. Para ello, habré de evaluar no sólo los conocimientos a los que ha llegado el alumno sino el progreso sufrido bonificando su nota por saltos en su conocimiento.

En definitiva, a este CIMA le falta un sistema de evaluación acorde a su modelo metodológico. Para ello me planteo tener en cuenta los siguientes criterios discutidos ya en Rivero y Porlan, 2017:

- Incorporar preguntas abiertas y estimulantes.

- Casos prácticos de situaciones cotidianas y profesionales de interés.

- Razonamientos de resultados obtenidos en prácticas o situaciones hipotéticas.

- Esquemas de procesos globales.

Respuestas que nos informarían de lo que realmente piensan los estudiantes y de la lógica que han utilizado para responder, poniendo así de manifiesto sus formas de razonar y la expresión de múltiples ideas relacionadas. Y, además, ya que no podríamos traducir en nota la evolución, este tipo de preguntas, nos permitirian calificar, que es una realidad que no podemos obviar, pues el alumno requiere una nota numérica para su expediente administrativo. 


\section{Conclusiones}

Es paradójico que nosotros que somos expertos en educación nos resulte tan dificil transmitir nuestro conocimiento haciendo único responsable al alumno de su no-aprendizaje. Esta visión autoritaria de la enseñanza, en la que se simplifica el funcionamiento del sistema neuronal y de la comunicación humana, ha perdurado siglos. Es normal que sea dificil salir de esa zona de confort para el docente evitando frustraciones y no haciéndose responsable del hecho de que el alumno no aprenda.

Enseñar y aprender es un proceso complejo, que requiere interacciones que necesitan planificación y un análisis riguroso. Para que esto ocurra en un contexto que me de seguridad, en mi clase, hay que desarrollar estrategias. Cada profesor debe hacer un autoanálisis riguroso de su enseñanza-aprendizaje y que quede recogido en un manual de ensayo-error como pretendemos hacer con estos Ciclos de Mejora en el Aula.

Jornadas de Formación e Innovación Docente del Profesorado | № 2 (2019) Esta obra se distribuye con la licencia Creative Commons 


\section{Referencias Bibliográficas}

ÁLVAREZ-MÉNDEZ, JM. (2007). Evaluar para conocer, examinar para excluir. Ediciones Morata. ISBN: 978-8471124623.

CURTIS, H, BARNES, S, SCHNEK, A, MASSARINI, A. (2008). Biología. 7ạ Edición Editorial Panamericana. ISBN: 978-950-06-0334-8.

DE ALBA, N, PORLÂN, R. La Metodología de Enseñanza. En R. Porlán y cols. (Eds.). Enseñanza Universitaria. Cómo mejorarla. pp 45-49. Madrid. Ediciones Morata.

FERIA, AB. (2017). La Fisiología Vegetal como Ciencia Integradora, una estrategia de enseñanza basada en la investigación. En R. Porlán y cols. (Eds.). Enseñanza Universitaria. Cómo mejorarla. pp 121-131. Madrid. Ediciones Morata.

GARCİA-DİAZ, E, PORLÁN, R, NAVARRO, E. (2017). Los fines y los contenidos de enseñanza. En R. Porlán y cols. (Eds.). Enseñanza Universitaria. Cómo mejorarla. pp 55-72. Madrid. Ediciones Morata.

RIVERO, A, PORLÂN, R. La evaluación en la enseñanza universitaria. En R. Porlán y cols. (Eds.). Enseñanza Universitaria. Cómo mejorarla. pp 73-91. Madrid. Ediciones Morata.

SÁNCHEZ-ROMERO, S, NAVARRO, E. (2018). Como trabajan los docentes con las ideas previas de los alumnos. Trabajo de Fin de Grado, Grado en Educación Primaria, Dpto. Didáctica de las Ciencias Experimentales y Sociales.

Travé, G, Pozuelo, F, Cañal, P. (2006). ¿Cómo enseñar investigando? Análisis de las percepciones de tres equipos docentes con diferentes grados de desarrollo profesional. Revista Iberoamericana de Educación. ISSN: 1681-5653.

Jornadas de Formación e Innovación Docente del Profesorado | № 2 (2019) Esta obra se distribuye con la licencia Creative Commons Reconocimiento-NoComercial-SinObraDerivada 